\title{
Robust Entrainment to Natural Oscillations of Asymmetric Systems arising from Animal Locomotion
}

\author{
Zhiyong Chen and Tetsuya Iwasaki
}

\begin{abstract}
We consider a class of linear flexible mechanical systems arising from the dynamics of animal locomotion. A distinctive property of such systems is that the stiffness matrix is asymmetric. Extending the standard notion to this class, we define the natural oscillation as a free response under the damping compensation to achieve marginal stability. As a benchmark, a link chain system in a fluid environment is considered, and its natural oscillation is shown to exhibit travelling waves appropriate for undulatory swimming. Moreover, we propose nonlinear feedback controllers, inspired by neuronal dynamics, to achieve entrainment to the natural oscillation.
\end{abstract}

Index Terms - Oscillations, locomotion, robotics, neuronal control, autonomous vehicles

\section{INTRODUCTION}

Diverse patterns are observed in rhythmic body movements during animal locomotion. It is conjectured that the energy consumption during locomotion is minimized by exploiting the mechanical resonance between the body and the surrounding environment [1], [2]. The idea of resonance exploitation will be useful for designing efficient robotic locomotors that are robust against and adaptive to environmental changes. However, underlying control mechanisms are largely unknown.

Resonance phenomena in standard ${ }^{1}$, lightly damped mechanical systems are well characterized. In particular, a natural mode of oscillation is defined to be a free response of the modified system obtained by removing all the damping effects to achieve marginal stability for sustained oscillation. This definition, however, does not directly apply to typical models of body-environment interactions during locomotion. The environmental forces on the body appear in the equation of motion as terms containing an asymmetric stiffness matrix, and simple removal of damping effects does not result in marginally stable systems. Hence, a new definition for natural oscillations is necessary to pursue the idea of resonance exploitation in locomotion control.

Z. Chen is with the School of Electrical Engineering and Computer Science, The University of Newcastle, Callaghan, NSW 2308, Australia zhiyong. chen@newcastle.edu.au

T. Iwasaki is with the Department of Mechanical and Aerospace Engineering, UCLA, LA, CA 90095, iwasaki@seas. ucla. edu

${ }^{1}$ By standard mechanical systems, we mean those described by symmetric positive definite mass, stiffness, and damping matrices.
Biological control mechanisms for animal locomotion are known to consist of neuronal circuits, called the central pattern generator (CPG) [3]. A CPG can be modeled as a nonlinear oscillator, and when placed in a feedback loop, provides a basic control architecture to achieve coordinated oscillations of engineered systems [4]-[6]. Within the CPG framework, feedback control laws to achieve entrainment to a resonance have been studied for standard mechanical systems [7]-[12]. In particular, references [12], [13] analytically showed how simple CPGs can be used to provide nonlinear damping and achieve a stable oscillation near a resonance.

In this paper, we shall first extend the notion of natural oscillations to mechanical systems with asymmetric stiffness matrices, arising from body-environment interactions during animal or robotic locomotion. It is shown that the system becomes marginally stable, thereby defining a natural oscillation, if the damping effect is adjusted by a proper amount, which is analytically characterized in terms of a generalized eigenvalue of the mass-stiffness matrix pair. We shall then define a class of nonlinear feedback controllers whose architecture is inspired by CPGs, and formulate a problem of designing a controller to achieve a natural oscillation for the closedloop system. The idea of multivariable harmonic balance (MHB) [14], [15] is used to characterize the design specification approximately, and systematic methods are proposed to find the controller parameters that satisfy the MHB condition exactly. An underwater link chain system is used to demonstrate that its natural oscillations give expected undulatory swimming gaits and that the proposed controllers are able to achieve such locomotion robustly against perturbations in mechanical parameters.

\section{NATURAL OSCILLATIONS OF SYSTEMS WITH ASYMMETRIC STIFFNESS MATRIX}

A. The class of mechanical systems

Consider the class of mechanical systems described by

$$
J \ddot{\theta}+D \dot{\theta}+K \theta=u, \quad \theta(t) \in \mathbb{R}^{n},
$$

where we impose the following:

\section{Assumption 1}

(a) $J, K \in \mathbb{R}^{n \times n}$ and $J=J^{\top}>0$. 
(b) $D=\mu J$ for some $\mu \in \mathbb{R}$.

(c) All the eigenvalues of $J^{-1} K$ are simple and have positive real parts.

Note that the stiffness matrix $K$ is not necessarily symmetric. The equation of motion captures the linearized body dynamics of a typical locomotion system that interacts with the environment continually. The stiffness matrix $K$ in (1) is typically given by $K=K_{o}+v \Lambda$ where $K_{o}$ is a symmetric positive definite matrix representing the body stiffness, and $v \Lambda$ is an asymmetric matrix representing the skewed stiffness arising from the locomotion at velocity $v$ relative to the environment. In this case, the system (1) is stable when $v$ is small. As $v$ gets larger, some of the characteristic roots move to the right half complex plane and the system becomes unstable.

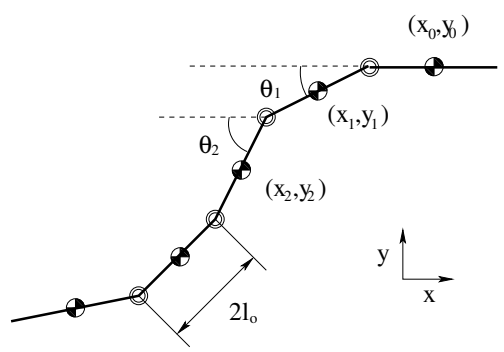

Fig. 1. Fliptail locomotor

As an example, consider a chain of multiple links connected by flexible joints, subject to environmental forces with directional preference, as shown in Fig. 1. The link at the right end (head) is constrained to slide along the $x$-axis so that $y_{o}(t) \equiv 0$. There are $n$ identical links in addition to the head, and each link has mass $m_{o}$, length $2 l_{o}$ and moment of inertia $m_{o} l_{o}^{2} / 3$, and each joint has torsional stiffness $k_{o}$. The angle between the $i^{\text {th }}$ link and the negative $x$-axis is denoted by $\theta_{i}$. Let $\tau_{i}$ be the torque applied at the $i^{\text {th }}$ joint. The environmental force on each link is modeled as $f_{n}=-\mu_{n} v_{n}$ and $f_{t}=-\mu_{t} v_{t}$, where $f_{n}$ and $f_{t}$ are the force components in the direction tangent and normal to the link, $v_{n}$ and $v_{t}$ are the components, in the respective directions, of the velocity of the link gravity center, and $\mu_{n}$ and $\mu_{t}$ are proportionality constants [16].

The equation of motion is given by (1) with

$$
\begin{aligned}
& J=m_{o} l_{o}^{2}\left(F F^{\top}+I / 3\right), \quad D=\left(\mu_{n} / m_{o}\right) J, \\
& K=v \Lambda+k_{o} B B^{\top}, \quad u=B \tau, \\
& \Lambda=\left(\mu_{n}-\mu_{t}\right) l_{o} F+\mu_{t} l_{o} \operatorname{diag}(F e)
\end{aligned}
$$

$B=\left[\begin{array}{cccc}1 & -1 & & \\ & \ddots & \ddots & \\ & & 1 & -1 \\ & & & 1\end{array}\right], F=\left[\begin{array}{cccc}1 & 2 & \cdots & 2 \\ & 1 & \ddots & \vdots \\ & & \ddots & 2 \\ & & & 1\end{array}\right]$,

where $e \in \mathbb{R}^{n}$ is the vector with all its entries being one, and $v:=\dot{x}_{o}$ is the head velocity. For the numerical examples given later, we use the following parameter values unless otherwise noted: The number of links for the tail is $n=5$, and each link has mass $m_{o}:=m / n$ and length $2 l_{o}=l / n$, where the total length is $l=0.5 \mathrm{~m}$ and mass is $m=0.2 \mathrm{~kg}$. The environmental force constants are $\mu_{t}=0$ and $\mu_{n}=0.2 \mathrm{Ns} / \mathrm{m}$, and each joint has stiffness $k_{o}=2.5 \times 10^{-4} \varkappa \mathrm{Nm} / \mathrm{rad}$, where the parameter $\varkappa$ is used to examine the effect of stiffness perturbation.

We call the system a fliptail locomotor since it is expected to maintain velocity $v$ by flipping (or undulating) the link chain. In this study, we gain insights into the locomotion mechanism by reversing the cause/effect and asking the following: if the head is forced to move at a constant velocity $v$, would the tail naturally tend to oscillate? If so, such natural oscillation might suggest a proper way to flip the tail to achieve efficient locomotion.

\section{B. Definitions of natural motions/oscillations}

Let us first recall some basic system concepts.

Definition 1 The characteristic equation of (1) is

$$
\left(\lambda^{2} J+\lambda D+K\right) z=0, \quad z \neq 0
$$

where $\lambda \in \mathbb{C}$ is an eigenvalue (or characteristic root) and $z \in \mathbb{C}^{n}$ is the associated mode shape. Suppose none of the eigenvalues are repeated. The free response of (1) for a particular initial condition described by $(\lambda, z)$ is given by a simple exponential function:

$u(t) \equiv 0, \theta(0)=\Re[z], \dot{\theta}(0)=\Re[\lambda z] \Rightarrow \theta(t)=\Re\left[z e^{\lambda t}\right]$.

The response $\theta(t)$ is called the natural motion.

There are $2 n$ natural motions for (1), and the free response to an arbitrary initial condition is given by a linear combination of the $2 n$ natural motions. A natural motion can also be described as

$$
\begin{array}{r}
\theta(t)=\Re\left[z e^{\lambda t}\right]=Z \cos (\omega t+\phi) e^{-\sigma t}, \\
Z e^{j \phi}:=z, \quad j \omega-\sigma:=\lambda,
\end{array}
$$

where $\phi \in \mathbb{R}^{n}, \omega, \sigma \in \mathbb{R}$, and $Z \in \mathbb{R}^{n \times n}$ is a diagonal matrix. $^{2}$ Thus, the natural motion associated with $(\lambda, z)$ is oscillatory if and only if the imaginary part of $\lambda$ is nonzero. In this case, the complex conjugate pair $(\bar{\lambda}, \bar{z})$ is also a solution to the characteristic equation since the coefficient matrices $J, D$, and $K$ are all real. It can readily be verified that the natural motion associated with $(\bar{\lambda}, \bar{z})$ coincides with that associated with $(\lambda, z)$. Hence, if there are exactly $m$ eigenvalues with nonzero imaginary part, then there are $n-m$ distinct natural motions.

\footnotetext{
${ }^{2}$ Throughout the paper, we use the notation $Z e^{j \phi}:=z$ to define $Z$ to be the diagonal matrix with $\left|z_{i}\right|$ on the $i^{\text {th }}$ diagonal entry, $\phi$ to be the vector with $\angle z_{i}$ in the $i^{\text {th }}$ entry, and $e^{j \phi}$ to be the vector with $e^{j \phi_{i}}$ stacked in a column.
} 
In the case of standard mechanical systems, a natural oscillation is defined to be a natural motion of the undamped system where $D$ is set to zero. We extend this concept for the class of mechanical systems arising from animal locomotion as follows.

Definition 2 Consider the system described by (1) with Assumption 1. Let the damping effect be adjusted by a parameter $\epsilon \in \mathbb{R}$ and define the modified system with no input:

$$
J \ddot{\theta}+(\mu-\epsilon) J \dot{\theta}+K \theta=0 .
$$

If this system has a nonzero characteristic root on the imaginary axis $\lambda= \pm j \omega$ with associated mode shape $z$ for a specific value $\epsilon:=\rho$, then the corresponding natural motion of (2) is called a natural oscillation $(\omega, z)$ of the original system (1) with damping factor $\rho$, where $\omega$ and $z$ are referred to as the natural frequency and mode shape of the natural oscillation.

When $J$ and $K$ are symmetric positive definite, this definition reduces to the standard notion of natural oscillations, where the damping factor is given by $\rho:=\mu$ and the choice $\epsilon:=\rho$ cancels the damping effect exactly. In this case, the system is marginally stable with all the eigenvalues on the imaginary axis. For the class of systems we consider, it is not obvious how to characterize $\rho$ nor how the system behaves under a choice of $\epsilon:=\rho$. The following section will provide some analytical results that help us understand the natural oscillations for our system.

\section{Analysis of natural oscillations}

Let us first characterize the set of all natural oscillations for (1).

Lemma 1: Consider the system in (1) with Assumption 1. Let $\omega, \rho \in \mathbb{R}$ and $z \in \mathbb{C}^{n}$ be given. Then, $(\omega, z)$ is a natural oscillation of (1) with damping factor $\rho$ if and only if $(\omega, z, \rho) \in \mathbb{N}$ where

$$
\begin{array}{r}
\mathbb{N}:=\left\{(\omega, z, \rho) \in \mathbb{R} \times \mathbb{C}^{n} \times \mathbb{R}: \omega=\sqrt{\Re(\varsigma)},\right. \\
\left.\rho=\mu+\frac{\Im(\varsigma)}{\sqrt{\Re(\varsigma)}}, \quad(\varsigma, z) \in \mathbb{M}\right\}
\end{array}
$$

and $\mathbb{M}$ is the set of generalized eigenvalue/eigenvector pairs of $(J, K)$ :

$$
\mathbb{M}:=\left\{(\varsigma, z) \in \mathbb{C} \times \mathbb{C}^{n}:(\varsigma J-K) z=0, z \neq 0\right\} .
$$

Since there are $n$ distinct elements in $\mathbb{M}$, there are $n$ natural oscillations for the system (1). It should be noted that the amplitude of each natural oscillation, $\|z\|$, is arbitrary.

Next, we characterize the stability property of (2).

Lemma 2: Consider the system (2), where $\mu, \epsilon \in \mathbb{R}$ and Assumption 1 (a) and (c) are satisfied. Let $\varrho$ be the smallest damping factor to which there corresponds a natural oscillation:

$$
\varrho:=\min _{(\varsigma, z) \in \mathbb{M}} \mu+\frac{\Im(\varsigma)}{\sqrt{\Re(\varsigma)}}
$$

and suppose the minimizer is unique. Then, the system is exponentially stable if $\varrho>\epsilon$, marginally stable if $\varrho=\epsilon$, and exponentially unstable if $\varrho<\epsilon$.

From Lemmas 1 and 2, we can conclude that the natural oscillation of (1) with damping factor $\varrho$ is stable but all the other natural oscillations are not. The following result precisely states this fact.

Lemma 3: Consider the system (1) with Assumption 1. Define $\varrho$ by (3) and suppose the minimizer is unique. Let $(\omega, z)$ be the natural oscillation of (1) with damping factor $\varrho$. For given $\epsilon$ and initial condition $\theta(0)$ and $\dot{\theta}(0)$, let $\theta(t)$ be the solution of (2). If $\epsilon=\varrho$, then there exist $\gamma, t_{o} \in \mathbb{R}$, dependent on the initial condition, such that

$$
\lim _{t \rightarrow \infty}\left|\theta(t)-\gamma Z \cos \left(\omega\left(t+t_{o}\right)+\phi\right)\right|=0, \quad Z e^{j \phi}:=z .
$$

Moreover, $\theta(t)$ globally converges to zero if $\epsilon<\varrho$, and diverges to infinity for almost all initial conditions if $\epsilon>\varrho$.

Example 1 The profiles of natural oscillations are calculated for the fliptail locomotor for several cases of locomotion velocity $v$ and joint stiffness $k_{o}$, and the result is summarized in Table I. For each case, the phase of the $i^{\text {th }}$ joint angle $\phi_{i}$ lags behind its anterior neighbor $\phi_{i-1}$, indicating travelling waves that propagate from head to tail. When $v_{o}=0.15 \mathrm{~m} / \mathrm{s}$, the snapshots with $\varkappa=0.5$ and $\varkappa=5$ are shown in Fig. 2. We see that more travelling waves are exhibited if the tail is softer.

TABLE I

NATURAL OSCILLATION VS $v$ AND $\varkappa$.

\begin{tabular}{|c|c||c|c|c|c|c|c|}
\hline$v(\mathrm{~m} / \mathrm{s})$ & $\varkappa$ & Period $(\mathrm{s})$ & $\phi_{1}$ & $\phi_{2}$ & $\phi_{3}$ & $\phi_{4}$ & $\phi_{5}$ \\
\hline 0.3 & 5 & 1.97 & $184^{\circ}$ & $122^{\circ}$ & $76^{\circ}$ & $20^{\circ}$ & $0^{\circ}$ \\
\hline 0.5 & 1 & 1.75 & $415^{\circ}$ & $290^{\circ}$ & $179^{\circ}$ & $80^{\circ}$ & $0^{\circ}$ \\
\hline 0.5 & 5 & 1.50 & $234^{\circ}$ & $154^{\circ}$ & $98^{\circ}$ & $35^{\circ}$ & $0^{\circ}$ \\
\hline
\end{tabular}

\section{Entrainment to NATURAL Oscillation}

In this section, we focus on the natural oscillation with the smallest damping factor $\varrho$, and will refer to it simply as the natural oscillation. In view of Lemma 3, we assume throughout this section that the minimizer in (3) is unique.

\section{A. Problem Formulation}

We would like to develop a systematic method for designing a feedback controller for (1) to achieve the natural oscillation with a prescribed amplitude as a stable limit cycle of the closed-loop system. Since the plant is linear, the controller is necessarily nonlinear to achieve structural stability of the limit cycle. The problem is difficult in general, and hence we will formulate a more 

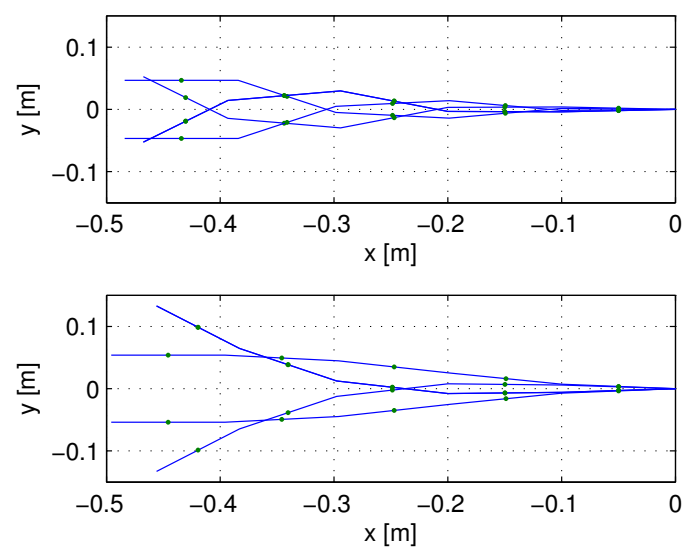

Fig. 2. Natural oscillation snapshots with different stiffness. Above: $\varkappa=0.5$; Below: $\varkappa=5$.

tractable problem whose solution practically solves the original problem. To this end, let us first fix the structure of the nonlinear controller, and then establish a set of control specifications that approximate the original problem in a certain sense.

Consider the damping augmentation by feedback:

$$
u=\epsilon J \dot{\theta}+w
$$

where $w$ is the new input after the augmentation. The idea is to choose $\epsilon<\varrho$ so that the augmented system is stable (Lemma 2), and then apply a sinusoidal input $w$ at the natural frequency $\omega$ with appropriate amplitudes and phases to drive the stable system so that the response converges to the natural oscillation with the prescribed amplitude in the steady state. The input $w$ is to be generated by a nonlinear feedback controller of the following form:

$$
w=G \psi(q), \quad q=f(s) H \theta
$$

where $G$ and $H$ are $n \times n$ real matrices, $f(s)$ is a scalar transfer function, and $\psi$ is a static nonlinearity satisfying the following properties:

- $\psi$ is odd, bounded, and strictly increasing.

- $\psi(x)$ is strictly concave on $x>0$, and $\psi^{\prime}(0)=1$.

For the rest of the paper, $\psi$ is a given function having these properties. The structure in (5) is motivated by biological control mechanisms. In particular, the simplest input-output model of neuronal dynamics is given by $v_{\text {post }}=\psi\left(f(s) v_{\text {pre }}\right)$ from the presynaptic potential $v_{\text {pre }}$ to the postsynaptic potential $v_{\text {post }}$ where $\psi$ and $f(s)$ represent the threshold nonlinearity and dynamics (time lag, adaptation, etc.) associated with synaptic and cell membrane processes, respectively. The controller in (5) is a network of multiple neurons with the interconnections specified by $G$ and $H$.
Let us now consider the closed-loop system formed by the plant (1) and the controller (4) and (5):

$$
\begin{array}{r}
P_{\epsilon}(s) \theta=G \psi(q), \quad q=f(s) H \theta \\
P_{\epsilon}(s):=s(s+\mu-\epsilon) J+K,
\end{array}
$$

where $s$ is the Laplace (or time derivative) operator. Suppose the system has a periodic solution that can be approximated by sinusoids:

$$
q(t)=\Re\left[\hat{q} e^{j \varpi t}\right], \quad \theta(t)=\Re\left[\hat{\theta} e^{j \varpi t}\right],
$$

where $\hat{q}, \hat{\theta} \in \mathbb{C}^{n}$ are the phasors of $q$ and $\theta$, respectively. We approximate the static nonlinearity by

$$
\psi(q) \cong \mathcal{K}(|\hat{q}|) q, \quad \mathcal{K}(x):=\operatorname{diag}\left(\kappa\left(x_{1}\right), \ldots, \kappa\left(x_{n}\right)\right)
$$

where $\kappa$ is the describing function of $\psi$. The multivariable harmonic balance condition is then given by

$$
P_{\epsilon}(j \varpi) \hat{\theta}=G \mathcal{K}(|\hat{q}|) \hat{q}, \quad \hat{q}=f(j \varpi) H \hat{\theta} .
$$

Solving the MHB condition for $(\varpi, \hat{\theta}, \hat{q})$, we have an estimate for an oscillatory trajectory of the closed-loop system as in (7). The MHB equation typically has multiple solutions, and thus predicts multiple oscillations. But some of them may be stable, and others may not be. For each MHB solution, the predicted oscillation is "expected" to be stable if the corresponding quasi-linear system is marginally stable [15]. The quasi-linear system is defined by replacing the nonlinearities in the closed-loop system with their describing functions evaluated at the predicted amplitudes.

Natural Entrainment Problem: Consider the system (1) and the controller described by (4) and (5), where $\psi$ is given and Assumption 1 holds. Let $(\omega, z)$ be the natural oscillation of (1) with damping factor $\varrho$, where $\|z\|$ is fixed as desired. Find the control gains $G, H \in \mathbb{R}^{n \times n}$ and transfer function $f(s)$ such that the following conditions are satisfied:

(i) The MHB equation (8) holds for $\varpi=\omega$ and $\hat{\theta}=z$.

(ii) The corresponding quasi-linearized system with $\psi(q)$ replaced by $\mathcal{K}(|f(j \omega) H z|) q$ is marginally stable.

We shall refer to $(\omega, z)$ with these two properties as a stable solution of (8).

\section{B. Feedback Control Design}

We first consider forced oscillations induced by a controller of the form (4). In particular, the natural oscillation for (1) can be achieved by stabilizing the system by the rate feedback $\epsilon J \dot{\theta}$ and applying a sinusoidal input $w$. The result can be summarized as follows.

Lemma 4: Consider the feedback system consisting of the plant (1) and controller (4), where Assumption 1 holds. Define $\varrho$ by (3) and let $(\omega, z, \varrho) \in \mathbb{N}$. Suppose

$w(t)=(\epsilon-\varrho) \omega J Z \sin (\omega t+\phi), \quad \epsilon<\varrho, \quad Z e^{j \phi}:=z$. 
Then, with an arbitrary initial condition, the trajectory $\theta(t)$ of the feedback system converges to the natural oscillation $(\omega, z)$ in the steady state.

Next, we shall address the Natural Entrainment Problem, considering the feedback controller described by (4) and (5). The natural oscillation $(\omega, z, \varrho) \in \mathbb{N}$ is expected to occur for the closed-loop system if the MHB equation (8) admits a solution $(\varpi, \hat{\theta}, \hat{q})$ with $\varpi=\omega$ and $\hat{\theta}=z$. Hence, we should choose the controller so that

$$
j \omega(\varrho-\epsilon) J z=G \mathcal{K}(|\hat{q}|) \hat{q}, \quad \hat{q}=f(j \omega) H z,
$$

where we used $P_{\epsilon}(j \omega) z=j \omega(\varrho-\epsilon) J z$. There are many choices for the dynamics $f(s)$ and gains $G$ and $H$ to satisfy this condition. For instance, the choice $H=Z^{-1}$ with $Z e^{j \phi}:=z$ would make the amplitudes of $\hat{q}_{i}$ uniform and simplify the equation to

$$
\begin{aligned}
&\left(\lambda_{\omega} J-G Z^{-1}\right) z=0, j \omega(\varrho-\epsilon) \\
& \hat{q}=f(j \omega) e^{j \phi}, \quad \lambda_{\omega}:=\frac{j(j \omega) \kappa(|f(j \omega)|)}{f(j)}
\end{aligned}
$$

Now, the condition is satisfied if

$$
\lambda_{\omega}=1, \quad G=J Z .
$$

Summarizing the result, we have the following.

Lemma 5: Consider the system (1) with Assumption 1. Let $(\omega, z)$ be the natural oscillation with damping factor $\varrho$ given by (3). Consider the controller

$$
u=\epsilon J \dot{\theta}+J Z \psi(q), \quad q=f(s) Z^{-1} \theta
$$

for given real number $\epsilon$, transfer function $f(s)$, and static nonlinearity $\psi$, where $Z e^{j \phi}:=z$. Suppose

$$
j \omega(\varrho-\epsilon)=\kappa_{\omega} f(j \omega), \quad \kappa_{\omega}:=\kappa(|f(j \omega)|)
$$

where $\kappa$ is the describing function of $\psi$. Then the $M H B$ equation (8) has a solution

$$
\varpi=\omega, \quad \hat{\theta}=z, \quad \hat{q}=f(j \omega) e^{j \phi} .
$$

Moreover, the associated quasi-linear system is marginally stable if and only if the set of $\lambda \in \mathbb{C}$ satisfying

$$
p(\lambda):=\lambda^{2}+(\mu-\epsilon) \lambda+\varsigma-\kappa_{\omega} f(\lambda)=0,
$$

for some generalized eigenvalue $\varsigma$ of $(J, K)$, is contained in the closed left half complex plane with no repetition on the imaginary axis.

If $(\varsigma, z) \in \mathbb{M}$, i.e., $\varsigma$ is the generalized eigenvalue of $(J, K)$ corresponding to the natural oscillation, then the characteristic equation (10) has a root $\lambda=j \omega$. To see this, first note from $(\omega, z, \varrho) \in \mathbb{N}$ that

$$
\begin{array}{r}
\lambda^{2}+(\mu-\varrho) \lambda+\varsigma=(\lambda-j \omega)(\lambda+\mu-\varrho+j \omega), \\
\varsigma=\omega^{2}+j \omega(\varrho-\mu) .
\end{array}
$$

Using (9), equation (10) can then be factored as

$$
\begin{array}{r}
p(\lambda)=(\lambda-j \omega)\left(\lambda+\mu-\epsilon+j \omega-\kappa_{\omega} g(\lambda)\right)=0 \\
g(\lambda):=\frac{f(\lambda)-f(j \omega)}{\lambda-j \omega},
\end{array}
$$

from which we conclude that $p(j \omega)=0$.

We now choose the controller dynamics $f(s)$ so that condition (9) is satisfied and the associated quasi-linear system is marginally stable. In view of Lemma 4 , it appears reasonable to choose $\epsilon$ less than $\varrho$ due to stability consideration. In this case, condition (9) requires that the phase angle of $f(j \omega)$ be $90^{\circ}$. The simplest choices for $f(s)$ to satisfy the phase property are $f(s)=\eta s$ and $f(s)=-\eta / s$ for some positive constant $\eta$. These choices are consistent with the previous result [12] that a CPG acts like a positive derivative feedback or a negative integral feedback when achieving entrainment to a resonance of a standard mechanical system. Below, we will formally show that these choices of $f(s)$ can indeed achieve entrainment to the natural oscillation of a mechanical system with an asymmetric stiffness matrix.

Theorem 1 (Positive derivative feedback): Consider the system (1) with Assumption 1. Let $(\omega, z)$ be the natural oscillation with damping factor @ given by (3). Let $\epsilon \in \mathbb{R}$ be such that

$$
0<\omega(\varrho-\epsilon)<1 .
$$

Then there exists a positive $\eta \in \mathbb{R}$ such that

$$
\varrho-\epsilon=\kappa(\eta \omega) \eta,
$$

where $\kappa$ is the describing function of $\psi$. For the controller

$$
u=\epsilon J \dot{\theta}+J Z \psi(q), \quad q=\eta Z^{-1} \dot{\theta}
$$

where $Z e^{j \phi}:=z$, the corresponding MHB equation (8) has a stable solution

$$
\varpi=\omega, \quad \hat{\theta}=z, \quad \hat{q}=j \omega \eta e^{j \phi} .
$$

The overall effect of the control $u$ is roughly equal to the linear control $\varrho J \dot{\theta}$ that achieves the natural oscillation. This is justified under the approximations of $\theta$ by sinusoids and $\psi$ by its describing function. However, the nonlinearity $\psi$ is expected to make the natural oscillation structurally stable. Note that $\kappa(x) x$ is a monotonically increasing function of $x$, approaching from zero to one when $x$ goes from zero to infinity. If $\epsilon \cong \varrho$, then $\eta \cong 0$ and convergence to the natural oscillation would be slow because $q$ is small and the effect of the nonlinearity $\psi$ is small, making the closed-loop system behave like linear systems lacking structural stability of periodic orbits. If $\epsilon \cong \varrho-1 / \omega$, then $\eta$ is very large and the convergence would be fast, exploiting the amplitude-dependent damping effect achieved by $\psi$. 
Example 2 Consider the fliptail locomotor with the positive derivative feedback. The resulting oscillation profiles of the closed-loop system are calculated from simulations and are listed in Table II, which is to be compared with the natural oscillations shown in Table I. The first row shows the result for the choice $Z=\operatorname{diag}(|z|)$ as described in Theorem 1. We see that entrainment to the natural oscillation is achieved approximately. In the second row, we modified the controller by using $Z=I$. The oscillation profile is slightly perturbed, but is still reasonably close to the natural oscillation. In the third and fourth rows, the same controller is used but the plant is perturbed. The resulting oscillations are remarkably close to the natural oscillations even under the perturbed conditions for which the controller is not designed. This example illustrate that (i) the controller has an inherent robustness to maintain the natural oscillation even if its profile changes due to system perturbations, and (ii) the control design does not require the precise values of the parameters $\left(v, k_{o}, \mu_{n}\right.$ and $\mu_{t}$ ) that determine the mode shape $z$.

TABLE II

OSCILLATION PROFILES WITH POSITIVE DERIVATIVE FEEDBACK

\begin{tabular}{|c|c|c||c|c|c|c|c|c|}
\hline$Z$ & $v$ & $\varkappa$ & Period & $\phi_{1}$ & $\phi_{2}$ & $\phi_{3}$ & $\phi_{4}$ & $\phi_{5}$ \\
\hline$|z|$ & 0.3 & 5 & 1.97 & $183^{\circ}$ & $120^{\circ}$ & $76^{\circ}$ & $20^{\circ}$ & $0^{\circ}$ \\
\hline $\mathrm{I}$ & 0.3 & 5 & 1.91 & $215^{\circ}$ & $140^{\circ}$ & $90^{\circ}$ & $26^{\circ}$ & $0^{\circ}$ \\
\hline $\mathrm{I}$ & 0.5 & 1 & 1.73 & $425^{\circ}$ & $298^{\circ}$ & $185^{\circ}$ & $82^{\circ}$ & $0^{\circ}$ \\
\hline $\mathrm{I}$ & 0.5 & 5 & 1.48 & $252^{\circ}$ & $174^{\circ}$ & $108 \circ$ & $41^{\circ}$ & $0^{\circ}$ \\
\hline
\end{tabular}

Theorem 2 (Negative integral feedback): Consider the system (1) with Assumption 1. Let $(\omega, z)$ be the natural oscillation with damping factor @ given by (3). Let $\epsilon \in \mathbb{R}$ be such that

$$
0<\omega(\varrho-\epsilon)<1 .
$$

Then there exists a positive $\eta \in \mathbb{R}$ such that

$$
\omega^{2}(\varrho-\epsilon)=\kappa(\eta / \omega) \eta,
$$

where $\kappa$ is the describing function of $\psi$. For the controller

$$
u=\epsilon J \dot{\theta}+J Z \psi(q), \quad \dot{q}=-\eta Z^{-1} \theta
$$

where $Z e^{j \phi}:=z$, the corresponding $M H B$ equation (8) has a solution

$$
\varpi=\omega, \quad \hat{\theta}=z, \quad \hat{q}=j(\eta / \omega) e^{j \phi} .
$$

Moreover, it is a stable solution if

$$
\omega^{2}=\min _{(\varsigma, z) \in \mathbb{M}} \Re(\varsigma) .
$$

The condition in (14) means that the generalized eigenvalue $\varsigma$ of $(J, K)$, that minimizes $\Im(\varsigma) / \sqrt{\Re(\varsigma)}$ as in (3) and characterizes the natural oscillation with the smallest damping factor $\varrho$, is the one having the smallest real part. Let such $\varsigma$ be denoted by $x_{o}+j y_{o}$. Then uniqueness of the minimizer of (3) implies that $y_{0}<0$. Moreover, condition (14) is satisfied if and only if all the generalized eigenvalues $x+j y$, other than $x_{o} \pm j y_{o}$, are in the region with parabolic and straight boundaries, specified by $\left|y / y_{o}\right|<\sqrt{x / x_{o}}$ and $x \geq x_{o}$.

\section{CONCLUSION}

We have considered a class of mechanical systems characterized by asymmetric stiffness matrices, arising from typical dynamics of animal locomotion. The natural oscillation is defined for such systems as a free response of damping-compensated systems, and is shown to capture rhythmic movements of flipping tails for swimming. Systematic methods are proposed for designing nonlinear feedback controllers to achieve entrainment to the natural oscillation. The control architectures are inspired by biological mechanisms of positive derivative feedback and negative integral feedback.

Acknowledgments: The work of the first author is supported by the Australian Research Council under grant No. DP0878724. The work of the second author is supported by the ONR MURI Grant N00014-08-1-0642, and by the NSF No.0654070.

\section{REFERENCES}

[1] G.A. Cavagna, N.C. Heglund, and C.R. Taylor. Mechanical work in terrestrial locomotion: Two basic mechanisms for minimizing energy expenditure. Am. J. Physiol., 233:R243-R261, 1977.

[2] J. Rose and J.G. Gamble. Human Walking. Lippincott Williams and Wilkins, 2003.

[3] G.N. Orlovsky, T.G. Deliagina, and S. Grillner. Neuronal Control of Locomotion: From Mollusc to Man. Oxford University Press, 1999.

[4] Y. Fukuoka, H. Kimura, and A.H. Cohen. Adaptive dynamic walking of a quadruped robot on irregular terrain based on biological concepts. Int. J. Robotics Research, 22(3-4):187-202, 2003.

[5] G. Taga. Self-organized control of bipedal locomotion by neural oscillators in unpredictable environment. Biol. Cybern., 65:147159, 1991.

[6] M.A. Lewis and G.A. Bekey. Gait adaptation in a quadruped robot. Autonomous Robots, 12(3):301-312, 2002.

[7] N.G. Hatsopoulos. Coupling the neural and physical dynamics in rhythmic movements. Neural Computation, 8(3):567-581, 1996.

[8] M.M. Williamson. Neural control of rhythmic arm movements. Neural Networks, 11:1379-1394, 1998.

[9] B.W. Verdaasdonk, H.F. Koopman, and F.C. Van der Helm. Energy efficient and robust rhythmic limb movement by central pattern generators. Neural Network, 19(4):388-400, 2006.

[10] B.W. Verdaasdonk, H.F. Koopman, and F.C. Van der Helm. Resonance tuning in a neuro-musculo-skeletal model of the forearm. Biological Cybernetics, 96(2):165-180, 2007.

[11] T. Iwasaki and M. Zheng. Sensory feedback mechanism underlying entrainment of central pattern generator to mechanical resonance. Biological Cybernetics, 94(4):245-261, 2006.

[12] Y. Futakata and T. Iwasaki. Formal analysis of resonance entrainment by CPG. J. Math. Biol., 57(2):183-207, 2008.

[13] Y. Futakata and T. Iwasaki. Entrainment of central pattern generators to neuronal oscillations of collocated mechanical systems. IEEE Conf. Decision and Contr., 2008.

[14] A. Gelb and W.E.V. Velde. Multiple-input describing functions and nonlinear system design. McGraw-Hill, New York, 1968.

[15] T. Iwasaki. Multivariable harmonic balance for central pattern generators. Automatica, 44(12):4061-4069, 2008.

[16] M. Saito, M. Fukaya, and T. Iwasaki. Serpentine locomotion with robotic snake. IEEE Control Systems Magazine, 22(1):64-81, 2002. 